\title{
TELEVISION Y BEBIDAS ALCOHÓLICAS Y ANALCOHÓLICAS
}

\author{
Pedro Naveillan F. * \\ Edith Cornejo A. * \\ Ilse López B. * \\ Heliette Saint - Jean **
}

\begin{abstract}
NAVEILlaN F., P. et al. Television y bebidas alcohólicas y analcohólicas. Rev. Saúde públ., S.Paulo, $21: 37-43,1987$.

RESUMEN: Dadas las implicancias educativas de la televisión, se decidió estudiar la frecuencia y características de los eventos con bebidas alcohólicas y analcohólicas en la televisión chilena utilizando el método ideado por Garlington. El registro fue hecho por períodos de media hora, distribuidos aleatoriamente, para cada observador, voluntaria perteneciente a una comuna que se caracteriza por su bajo nivel sociocultural; ellas monitorearon durante una semana, de Lunes a Viemes todos los programas transmiti. dos desde las 20:00 a las 24:00 horas. Se observó que, en promedio, por cada canal se transmite un evento de bebida alcohólica cada 24 minutos 19 segundos y uno de bebida no alcohólica cada 37 minutos y medio; las imágenes de bebida alcohólicas se incrementan a partir de las 21:30 horas. Del total de eventos, $60,7 \%$ corresponden a bebidas alcohólicas y de éstos el $61,1 \%$ tiene carácter publicitario. Los eventos relacionados con bebidas alcohólicas ocurren principalmente en la casa, por amistad y en forma de escenas, los con no alcohólicas en lugares desportivos, por amistad y en escena de ingestión.
\end{abstract}

DESCRIPTORES: Bebidas alcohólicas. Alcoholismo, prevenção e controle. Bebidas. Television. Educacion en salud.

\section{INTRODUCCION}

Es un hecho universalmente reconocido que en la prevención primaria del alcoholismo y de los problemas derivados del alcohol, la educación para la salud es uno de los pilares ecenciales; ésta se da en un contexto sociocultural que no puede ser desconocido y en cierto modo propone cambios respecto a las actitudes y los hábitos de consumo de las bebidas alcohólicas.

Dado que la televisión tiene una significación educativa, positiva o negativa, que crea y modifica actitudes y conductas, se hace importante conocer qué mensajes está recibiendo la población a través de este medio audiovisual que facilita o dificulta la educación para la salud en materias de alcohol y al. coholismo.

En Estados Unidos, se ha estudiado a fondo el impacto que puede tener la televisión ${ }^{1}$ como medio educativo. Igualmente se ha analizado en ex. tenso la relación entre la violencia en la comunidad y en los programas de TV.

Ultimamente ha aparecido un interesante método de estudio que permite cuantificar los mensajes que la televisión envía respecto del consumo de bebidas, particularmente alcohólicas ${ }^{2,3,4}$.

Ha parecido de interés adaptar esta metodología y sus instrumentos al medio nacional chileno. El presente estudio es el primer intento en el proceso de esta adaptación, sus objetivos de caracter explo. ratorio son:

- Medir la frecuencia de aparición de eventos relacionados con bebidas alcohólicas y no alcohólicas en los programas de televisión de Santiago y describir su presentación con algunas variables de las mismas. Tales como tipo de programa (publicidad - no publicidad), modo de aparición (referencia verbal, escena de ingestión, situación de fondo), lugar de aparición: bar, casa, club desportivo, otro no especificado), razón para ingerir (relajación, hospitalidad, celebración, no hay razón).

La investigación se pudo realizar gracias a la colaboración de la Fundación Educacional San Pablo, con la que se está trabajando un programa intracomunitario de tipo educativo de prevención de los problemas del alcohol y alcoholismo.

\section{METODOLOGIA}

Se seleccionaron los tres canales de TV de la Re. gión Metropolitana que se designaron como A, B y C, y se observaron todos los programas transmitidos en ellos desde las 20:00 a las 24:00 horas, de Lunes a Viemes, durante una semana, elegida al azar la cual resutó ser tercera del mes de Enero de 1984; para el registro de los eventos relacionados con bebi-

\footnotetext{
- Escuela de Salud Publica. Universidad de Chile - Independencia No 939 - Casilla 6537 - Correo 7 - Santiago - Chile.

- Facultad de Medicina. Universidad de Chile. División Oriente - Santiago - Chile.
} 
das que pudieran aparecer en ellos se utilizó el instrumento diseño por Garlington ${ }^{2}$, el cual fue traducido al castellano.

Se contemplaron periodos de observación de media hora de duración, dividiendo el lapso a observar en unidades de este tamaño, las cuales se distribuyeron aleatoriamente para los efectos de asignar a cada observador los espacios correspondientes.

Se utilizó como observadores a doce voluntarias de la Comuna de Pudahuel que colaboran con la Fundación San Pablo, en cuyas escuelas tienen sus hijos; la mayoría de ellos habian seguido previamente un curso en la Escuela de Salud Pública, de capacitación como líderes para actuar en la prevención de los problemas de alcohol y alcoholismo.

Para los efectos de este trabajo fueron capacitadas específicamente en la utilización del instrumento, el que fue probado dos veces, observando todas el mismo programa de TV hasta que se obtuvo una coincidencia sobre el $85 \%$ y que juzgamos aceptable.

E1 instrumento contempla registros de los even. tos que ocurren minuto a minuto y variables que dicen relación con el tipo de bebida; alcohólica y analcohólica; el modo de aparición: como re ferencia verbal, escenas de ingestión, situación de fondo; en lugar en que aparecen o se beben: bar, casa, club desportivo, otros; la razón de consumo: relajación, hospitalidad, amistad, celebración o no hay razón; y si trata de eventos publicitarios o no publicitarios (eventos que aparecen en el interior de los programas). Todas las variables se definieron operacionalmente transformándolas en indicadores factibles de observar y medir directamente por las observadoras: referencia verbal = invitación oral a beber; escena de ingestión en un primer plano, situación de fondo: imagen que no aparece en primer plano y no corresponde a las dos alternativas anteriores.

Los registros fueron revisados y elaborados ma. nualmente, con la metodología estadística descriptiva, sin análisis de significación por tratarse de un estudio exploratorio.

\section{RESULT ADOS}

\section{Numero y Distribucion de Eventos}

Se presentan el número de eventos, por períodos de media hora, durante el lapso de observación en que aparecen bebidas alcohólicas y no alcohólicas. Hubo un total de 148 eventos de bebidas alcohólicas y 96 de analcohólicas entre los tres canales, lo que significa que en promedio se presentó por canal uno de bebidas alcohólicas cada poco menos de $25 \mathrm{~min}$. (24m y 19s) y uno de no alcohólicas cada treinta y siete minutos y medio.

Los eventos correspondientes a bebidas alcohólicas se incrementan notoriamente a partir de las 21:30 h (lo que era dable de esperar por cuanto a partir de esa hora está autorizada la publicidad de este tipo de bebidas) encontrándo-se más de la cuarta parte de ellos entre las $23: 30$ y las $24: 00 \mathrm{~h}$ $(28,4 \%)$. En cambio los eventos de las bebidas no alcohólicas tienen su máxima frecuencia entre las $20: 00$ y las $20: 30 \mathrm{~h}(20,0 \%)$.

TABLA 1

Proporcion de eventos con bebidas alcohólicas y no alcohólicas en el lapso de observacion en 3 canales de TV, Santiago, Chile, enero 1984

\begin{tabular}{|c|c|c|c|c|c|c|c|}
\hline \multirow[b]{2}{*}{ Hora } & \multirow{2}{*}{$\begin{array}{c}\text { Tipo de } \\
\text { Bebida }\end{array}$} & \multicolumn{2}{|c|}{ Alcohólicas } & \multicolumn{2}{|c|}{ No Alcohólicas } & \multicolumn{2}{|c|}{ Total } \\
\hline & & $\overline{\mathrm{N}}$ & $\%$ & No & $\%$ & N! & $\%$ \\
\hline $20: 00$ & $-20: 30$ & 8 & 5,4 & 25 & 26,0 & 33 & 13,5 \\
\hline $20: 30$ & $-21: 00$ & 2 & 1,3 & 3 & 3,1 & 5 & 2,0 \\
\hline $21: 00$ & $-21: 30$ & 1 & 0,7 & 10 & 10,4 & 11 & 4,5 \\
\hline $21: 30$ & $-22: 00$ & 27 & 18,3 & 9 & 9,4 & 36 & 14,7 \\
\hline $22: 00$ & $-22: 30$ & 18 & 12,2 & 16 & 16,7 & 34 & 13,9 \\
\hline $22: 30$ & $-23: 00$ & 23 & 15,5 & 16 & 16,7 & 39 & 16,0 \\
\hline $23: 00$ & $-23: 30$ & 27 & 18,2 & 11 & 11,5 & 38 & 15,6 \\
\hline $23: 30$ & $-24: 00$ & 42 & 28,4 & 6 & 6,2 & 48 & 19,7 \\
\hline \multicolumn{2}{|c|}{ Total } & 148 & 100,0 & 96 & 100,0 & 244 & 100,0 \\
\hline
\end{tabular}

Se aprecia que la proporción de eventos entre los dos tipos de bebidas se invierte a partir de las 21:30 horas, predominando notoriamente los correspon. dientes a las bebidas alcohólicas, con $87,5 \%$ de ellos al término del periodo de observación. Respecto del total de eventos, el $60,7 \%$ corresponde a las bebidas alcohólicas. 
TABLA 2

Aparicion de los dos tipos de bebidas en cada $1 / 2$ hora de observacion en 3 canales de TV, Santiago, Chile, enero 1984.

\begin{tabular}{|c|c|c|c|c|c|c|}
\hline \multirow[t]{2}{*}{$\begin{array}{l}\text { Tipo de } \\
\text { Bebida }\end{array}$} & \multicolumn{2}{|c|}{ Alcohólica } & \multicolumn{2}{|c|}{ No Alcohólica } & \multicolumn{2}{|c|}{ Total } \\
\hline & $\overline{\mathrm{N} O}$ & $\overline{\%}$ & $\overline{\mathrm{N} Q}$ & $\%$ & No & $\overline{\%}$ \\
\hline $20: 00$ & 8 & 24,2 & 25 & 75,8 & 33 & 100,0 \\
\hline $20: 30$ & 2 & 40,0 & 3 & 60,0 & 5 & 100,0 \\
\hline $21: 00$ & 1 & 9,1 & 10 & 90,9 & 11 & 100,0 \\
\hline $21: 30$ & 27 & 75,0 & 9 & 25,0 & 36 & 100,0 \\
\hline $22: 00$ & 18 & 52,9 & 16 & 47,1 & 34 & 100,0 \\
\hline $22: 30$ & 23 & 59,0 & 16 & 41,0 & 39 & 100,0 \\
\hline $23: 00$ & 27 & 71,1 & 11 & 28,9 & 38 & 100,0 \\
\hline $23: 30$ & 42 & 87,5 & 6 & 12,5 & 48 & 100,0 \\
\hline Total & 148 & 60,7 & 96 & 39,3 & 244 & 100,0 \\
\hline
\end{tabular}

Nota: Las porcentages corresponden a las cifras de cada línea.

\section{Eventos y Publicidad}

Del total de eventos, el $61,1 \%$ tiene un carácter publicitario y el $38,9 \%$ corresponde a situaciones que se dan en el interior de otros programas o activi- dades. Lo publicitario es casi un $10,0 \%$ mayor en el caso de las bebidas no alcohólicas; y lo no publicitario es poco más de un $16,0 \%$ mayor en el caso de las bebidas alcohólicas.

TABLA 3

Eventos segun publicidad y tipo de bebida en los 3 canales de TV, Santiago, Chile, enero 1984.

\begin{tabular}{|c|c|c|c|c|c|c|}
\hline \multirow{2}{*}{ Tipo Bebida } & \multicolumn{2}{|c|}{ Publicidad } & \multicolumn{2}{|c|}{ No Publicidad } & \multicolumn{2}{|c|}{ Total } \\
\hline & $\overline{\mathrm{N} \varphi}$ & $\overline{\%}$ & No & $\%$ & $\overline{N o}$ & $\%$ \\
\hline Alcohólica & 87 & 58,8 & 61 & 41,2 & 148 & 100,0 \\
\hline No Alcohólica & 62 & 64,6 & 34 & 35,4 & 96 & 100,0 \\
\hline Total & 149 & 61,1 & 95 & 38,9 & 244 & 100,0 \\
\hline
\end{tabular}

Nota: Las porcentages corresponden a las cifras de cada línea.

Se presentan respectivamente los eventos de publicitarios de bebidas alcohólicas se concentran bebidas alcohólicas según publicidad y no publici- en el canal A $(58,6 \%)$ seguido por el canal C $(36,8 \%)$ dad, por canal y por unidad de tiempo. Los eventos siendo su presencia casi insignificante en el Canal

TABLA 4

Eventos de publicidad ce bebidas alcohólicas por canal de TV segun unidad de tiempo Santiago, Chile, enero, 1984.

\begin{tabular}{|c|c|c|c|c|c|c|c|c|c|c|}
\hline \multirow[b]{2}{*}{ Hora } & \multirow{2}{*}{\multicolumn{2}{|c|}{ Canal }} & \multicolumn{2}{|c|}{ A } & \multicolumn{2}{|c|}{$\mathrm{B}$} & \multicolumn{2}{|c|}{ C } & \multicolumn{2}{|c|}{ Total } \\
\hline & & & $\overline{\mathrm{N}}$ & $\%$ & $\overline{\mathrm{N}}$ & $\overline{\%}$ & $\overline{\text { No }}$ & $\overline{\%}$ & No & $\%$ \\
\hline $20: 00$ & - & $20: 30$ & - & & 1 & & - & & 1 & \\
\hline $20: 30$ & - & $21: 00$ & 2 & & - & & - & & 2 & \\
\hline $21: 00$ & - & $21: 30$ & - & & - & & - & & & \\
\hline $21: 30$ & - & $22: 00$ & 19 & & - & & 6 & & 25 & \\
\hline $22: 00$ & - & $22: 30$ & 4 & & 1 & & 4 & & 9 & \\
\hline $22: 30$ & - & $23: 00$ & 3 & & - & & 4 & & 7 & \\
\hline $23: 00$ & - & $23: 30$ & 5 & & - & & 3 & & 8 & \\
\hline $23: 30$ & - & $24: 00$ & 18 & & 2 & & 15 & & 35 & \\
\hline & Tots & & 51 & 58,6 & 4 & 4,6 & 32 & 36,8 & 87 & 100,0 \\
\hline
\end{tabular}


B $(4,6 \%)$. La preferencia horaria es por las $23: 30 \mathrm{~h}$, seguida por las $21: 30$ a 22:00 h. En cambio, la distribución no publicitaria es igual en los canales B y
C ( $36,0 \%)$ y muy parecida en el $\mathrm{A}(27,9 \%)$ con una prefe rencia horaria entre las $22: 30$ y $23: 30 \mathrm{~h}$.

TABLA 5

Eventos con bebidas alcohólicas en programas no publicitarios por canal segun unidad de tiempo, Santiago, Chile, enero, 1984.

\begin{tabular}{|c|c|c|c|c|c|c|c|c|c|c|}
\hline \multirow{2}{*}{ Hora } & \multirow{2}{*}{\multicolumn{2}{|c|}{ Canal }} & \multicolumn{2}{|c|}{ A } & \multicolumn{2}{|c|}{ B } & \multicolumn{2}{|c|}{ C } & \multicolumn{2}{|c|}{ Total } \\
\hline & & & No & $\overline{\%}$ & No & $\overline{\%}$ & $\overline{\mathrm{N} Q}$ & $\%$ & No & $\%$ \\
\hline $20: 00$ & - & $20: 30$ & 3 & & 1 & & 3 & & 7 & \\
\hline $20: 30$ & - & $21: 00$ & - & & - & & - & & - & \\
\hline $21: 00$ & - & $21: 30$ & - & & - & & 1 & & 1 & \\
\hline $21: 30$ & - & $22: 00$ & 1 & & - & & 1 & & 2 & \\
\hline $22: 00$ & - & $22: 30$ & 4 & & - & & 5 & & 9 & \\
\hline $22: 30$ & - & $23: 00$ & 8 & & 4 & & 4 & & 16 & \\
\hline $23: 00$ & - & $23: 30$ & 1 & & 12 & & 6 & & 19 & \\
\hline $23: 30$ & - & $24: 00$ & - & & 5 & & 2 & & 7 & \\
\hline & Tota & & 17 & 27,9 & 22 & 36,0 & 22 & 36,0 & 61 & 99,9 \\
\hline
\end{tabular}

\section{Eventos, publicidad y canales de television}

En los canales $\mathrm{A}$ y $\mathrm{C}$ los eventos publicitarios predominantes en relación con las bebidas alcohólicas, en el B, en cambio, con las no alcohólicas. Globalmente, prima la publicidad para las bebidas alcohóli. cas $(58,4 \%)$ sobre las no alcohólicas $(41,6 \%)$.

\section{TABLA 6}

Eventos publicitarios de bebidas alcohólicas y no alcohólicas por cada canal,

Santiago, Chile, enero, 1984

\begin{tabular}{cccccccc}
\hline \multirow{2}{*}{ Canal } & \multicolumn{2}{c}{ Alcohólicas } & & \multicolumn{2}{c}{ No Alcohólicas } & & \multicolumn{2}{c}{ Total } \\
\cline { 2 - 6 } & N! & $\%$ & & No & $\%$ \\
\hline A & 51 & 71,8 & 20 & 28,2 & 71 & 100,0 \\
B & 4 & 23,5 & 13 & 76,5 & & 17 & 100,0 \\
C & 32 & 52,5 & 29 & 47,5 & & 61 & 100,0 \\
\hline Total & 87 & 58,4 & 62 & 41,6 & & 149 & 100,0 \\
\hline
\end{tabular}

Nota: Las porcentages corresponden a las cifras de cada línea

En la situaciones no publicitarias, la aparición de even tos relacionados con bebidas alcohólicas $(64,2 \%)$ tiende también a predominar sobre los relacionados con bebidas no alcohólicas $(35,8 \%)$. Esto es particularmente cierto para el canal B en el que la aparición de bebidas alcohólicas en eventos no publicitarios al $88,0 \%$.

En síntesis: Los eventos relativos a bebidas alcohólicas son más frecuentes que los que dicen relación con las analcohólicas, haya o no de por medio publicidad.

\section{Eventos y modo de aparición}

Se observa que predominan las escenas de la ingestion $(66,4 \%)$ con igual frecuencia para ambos tipos de bebidas, en cambio, las situaciones de fondo son más frecuentes para las no alcohólicas $(24,0 \%$ vs $16,2 \%)$ y las referencias verbales para las alcoholicas $(17,6 \%$ vs $9,4 \%)$. En ésta última categoría es donde se encuentra la máxima diferencia favorable a las medidas alcohólicas.

\section{Bebidas y lugar de aparición o ingestión}

En los eventos que dan en el bar o en la casa, más de $70,0 \%$ corresponden a bebidas alcohólicas mientras que en los que se dan en relación con deportes u otros la distribución es similar para ambos tipos de bebidas; en la categoria no especificado se encuentra el $6,6 \%$ de los eventos.

La máxima frecuencia de los eventos con bebidas alcohólicas corresponde a lugares deportivos $u$ otros (60), igual que para las no alcohólicas (66). 
TABLA 7

Eventos no publicitarios de bebidas alcohólicas y no alcohólicas por cada canal, Santiago, Chile, enero, 1984.

\begin{tabular}{|c|c|c|c|c|c|c|}
\hline \multirow{2}{*}{ Canal } & \multicolumn{2}{|c|}{ Alcohólicas } & \multicolumn{2}{|c|}{ No Alcohólicas } & \multicolumn{2}{|c|}{ Total } \\
\hline & N! & $\%$ & $\overline{\text { No }}$ & $\%$ & $\overline{\mathrm{N}}$ & $\overline{\%}$ \\
\hline A & 17 & 54,8 & 14 & 45,2 & 31 & 100,0 \\
\hline B & 22 & 88,0 & 3 & 12,0 & 25 & 100,0 \\
\hline C & 22 & 56,4 & 17 & 43,6 & 39 & 100,0 \\
\hline Total & 61 & 64,2 & 34 & 35,8 & 95 & 100,0 \\
\hline
\end{tabular}

Nota: Las porcentages corresponden a las cifras de cada línea.

TABLA 8

Imagenes de bebidas alcohólicas y no alcohólicas segun modo de aparicion, Santiago, Chile, enero, 1984.

\begin{tabular}{|c|c|c|c|c|c|c|}
\hline \multirow{2}{*}{$\begin{array}{l}\text { Tipo de } \\
\text { Bebida } \\
\text { Modo de } \\
\text { Aparicion }\end{array}$} & \multicolumn{2}{|c|}{ Alcohólicas } & \multicolumn{2}{|c|}{ No Alcohólicas } & \multicolumn{2}{|c|}{ Total } \\
\hline & № & $\%$ & No & $\%$ & No & $\%$ \\
\hline $\begin{array}{l}\text { Referencia } \\
\text { Verbal }\end{array}$ & 26 & 17,6 & 9 & 9,4 & 35 & 14,3 \\
\hline Escena & 98 & 66,2 & 64 & 66,6 & 162 & 66,4 \\
\hline $\begin{array}{l}\text { Situación } \\
\text { Fondo }\end{array}$ & 24 & 16,2 & 23 & 24,0 & 47 & 19,3 \\
\hline Total & 148 & 100,0 & 96 & 100,0 & 244 & 100,0 \\
\hline
\end{tabular}

TABLA 9

Tipo de bebida y lugar de aparicion o ingestion, Santiago, Chile, enero, 1984.

\begin{tabular}{|c|c|c|c|c|c|c|}
\hline \multirow{2}{*}{$\begin{array}{lr} & \text { Tipo de } \\
\text { Bebida } & \\
\text { Lugar } \quad\end{array}$} & \multicolumn{2}{|c|}{ Alcohólicas } & \multicolumn{2}{|c|}{ No Alcohólicas } & \multicolumn{2}{|c|}{ Total } \\
\hline & $\overline{\mathrm{N} !}$ & $\%$ & N? & $\%$ & $\overline{\mathrm{No}}$ & $\%$ \\
\hline Bar & 19 & 76,0 & 6 & 24,0 & 25 & 100,0 \\
\hline Casa & 55 & 71,4 & 22 & 28,6 & 77 & 100,0 \\
\hline Club Detvo, Otro & 60 & 47,6 & 66 & 52,4 & 176 & 100,0 \\
\hline No especificado & 14 & 87,5 & 2 & 12,5 & 16 & 100,0 \\
\hline Total & 148 & 60,7 & 96 & 39,3 & 244 & 100,0 \\
\hline
\end{tabular}

Nota: Las porcentages corresponden a las cifras de cada línea.

\section{Eventos y razones para su consumo}

Este puede ser considerado uno de los puntos débiles de este estudio exploratorio ya que por los resultados, podria pensarse que no se logró operacionalizar lo suficiente cada dimensión en que fue categorizada la variable "razón para beber" (categorías que aparecen en la Tabla 10), no obstante, es interesante presentar-los, pues refleja cómo ha percebido el mensaje el grupo de observadoras voluntarias. En casi un tercio de los eventos no quedó clara la razón, lo qual significa que la escena solo mostraba su consumo pero no se evidenciaba la razón que las personas tenían para hacerlo (categoría, no hay razón). En algo más del $40 \%$ la razón para beber ambos tipos de bebidas es la amistad/hospitalidad, la celebración se presenta en el $18,9 \%$ de los eventos con bebidas alcohólicas y en el 5,2\% en las no alcohólicas; y la relajación en el $9,5 \%$ y $16,7 \%$, respectivamente. 


\section{TABLA 10}

Razon aparente para ingerir bebidas alcohólicas y no alcohólicas Santiago, Chile, enero, 1984.

\begin{tabular}{|c|c|c|c|c|c|c|}
\hline \multirow{2}{*}{$\begin{array}{r}\text { Tipo de } \\
\text { Bebida } \\
\text { Razon }\end{array}$} & \multicolumn{2}{|c|}{ Alcohólicas } & \multicolumn{2}{|c|}{ No Alcohólicas } & \multicolumn{2}{|c|}{ Total } \\
\hline & No & $\%$ & Nọ & $\%$ & No! & $\overline{\%}$ \\
\hline Relajación & 14 & 9,5 & 16 & 16,7 & 30 & 12,3 \\
\hline Hospitalidad & 61 & 41,2 & 41 & 42,7 & 102 & 41,8 \\
\hline Celebración & 28 & 18,9 & 5 & 5,2 & 33 & 13,5 \\
\hline No hay Razón & 45 & 30,4 & 34 & 35,4 & 79 & 32,4 \\
\hline Total & 148 & 100,0 & 96 & 100,0 & 244 & 100,0 \\
\hline
\end{tabular}

\section{Cruzamiento de Variables}

Cruzamos las variables lugar y razón, razón y modo de aparición, y lugar y modo de aparición, pero, obtuvimos muchos casilleros en blanco o con escassos números de eventos en ellos; igualmente la complejidad de la materia y el discutible valor de la información, dadas las dificultades señaladas de operacionalizar las variables, nos impide llegar a conclusiones, a lo más nos permite señalar algunas tendencias, que son: - los eventos relacionados con bebidas alcohólicas ocurren preferentemente en la casa, como manifestación de hospitalidad/amistad $\mathrm{y}$ en forma de escenas de ingestión; - los de bebidas no alcohólicas en lugares deportivos u otros y secundariamente en la casa y también como manifestación de amistad/hospitalidad y en forma de escenas de ingestión.

\section{DISCUSION}

Queremos remarcar que el nuestro es un estudio exploratorio en el que hemos tratado de adaptar una metodología norteamericana a nuestra realidad.

Las principales dificultades han tenido relación, igual que las de los investigadores norteamericanos relatan, con la operacionalización de variables y de preparación de los observadores. Es indudable que ambos aspectos deben ser refinados para dar mayor confiabilidad a los datos obtenidos.

Una segunda limitante de este trabajo proviene de que sólo se observó un período horário limitado de los programas de TV en las horas en que se concentra la publicidad de las bebidas alcohólicas. Es altamente probable que si se tomara todos los horarios, las situaciones no publicitarias de consumo de bebidas alcohólicas, predominarían sobre las publicitarias, como se vio en el trabajo de Garlington y col. $^{2}$

También ha introducido un sesgo el factor estacional puesto que es sabido en los me dios televisivos que el mes de Enero es uno de los menos representa. tivos y que seguramente la presencia de bebidas alcohólicas es mayor en otros periodos.

Conviene retener, además, que lo que se ha medido es la presentación de eventos con bebidas alcohólicas y no alcohólicas y nó su efecto sobre la teleaudiencia. Si bien hay diferentes trabajos que señalan el impacto educativo de la TV y su capacidad de moldear la conducta de las personas, éstos son estudios extranjeros que deberían ser duplicados en nuestro medio. Con todo, tanto los modos de ingestión como el lugar en que aparecen o se deben $y$ las razones inferidas para hacerlo, se relacionan con estilos de vida con los que el televidente se puede identificar $y$, cuando esto no es así, lo estimulan a desearlo.

\section{CONCLUSIONES}

1. Es posible cuantificar la aparición y contexto en que se dan las bebidas alcohólicas y analcohólicas en la TV.

2. En promedio se presenta por canal una imagen de bebida alcohólica cada $(24 \mathrm{~m} \mathrm{19s})$ y una de bebi. da no alcohólica cada $371 / 2 \mathrm{~min}$., en los programas de TV estudiados en esta investigación.

3. Los eventos con bebidas alcohólicas se incrementan notoriamente a partir de las 21:30 h.

4. El $60,7 \%$ del total de eventos cuantificados corresponden a bebidas alcohólicas de la cuales algo más de la mitad tienen carácter publicitario.

5. La distribución de los eventos con bebidas alcohólicas es desigual en los distintos canales.

6. Predominan los eventos de bebidas alcohólicas en escenas de ingestión en la casa como manifestación de hospitalidad o amistad y de bebidas no alco. hólicas en lugares deportivos u otros, también como manifestación de amistad y en escenas de ingestión. 
NAVEILLAN F., P. et al. [Television and drinking-alcoholic and nonalcoholic]. Rev.Saúde públ., S. Paulo, $21: 37-43,1987$.

ABSTRACT: In view of the educational impact of television, the frequency and characteristics of T.V. events associated both with alcoholic and non-alcoholic beverages were studied, according to Garlington's technique. The events in all the programs from $8 \mathrm{p} . \mathrm{m}$. to 12 midnight, Monday through Friday, "were registered by volunteers from an area of low socioeconomic status, in probabilisticably selected half an hour periods during a normal week. On the average the channels transmit an alcohol-related event every 24'19", and one non-alcoholic beverage associated event every 37'30". Alcohol drinking appears more often ofter $9: 30$ p.m. Of the total number of events, $60.7 \%$ are related to alcohol, $61.1 \%$ of these being advertisements. They take place mainly in the home or in a friendly atmosphere, under the guise of plays; non-alcoholic beverages were shown at sporting contexts, associated with friendship, and in drinking scenes.

UNITERMS: Alcoholic beverages. Alcoholism, prevention and control. Beverages. Television. Health education.

\section{REFERE๋NCIAS BIBLIOGRÁFICAS}

1. COELHO, G.V., ed. Television as a teacher. Bethesda, M.D., National Institute of Mental Health, 1981.

2. GARLINGTON, W.K. Drinking on television: a preliminary study with enphasis on method. J. Stud. Al. cohol., 38: 2199-205, 1977.

3. CAPISO, J.; GOODSBADT, M.; GARLINGTON, W.K. \& SHEPPARD, M. Television portrayal of alcohol and other beberages. J.Stud.Alcohol., 43 : $1232-43,1982$.
4. STRICKLAND, D.; FINN, A. \& LAMBERT, M.D. A content analysis of beberage alcohol adversiting. I magazine adversiting. J. Stud.Alcohol., 43:655-82, 1982.

Recebido para publicaçāo em 03/04/1986

Reopresentado em 30/09/1986

Aprovado para publicação em 11/11/1986 